\title{
Confiabilidade das medidas de espessura central da córnea com Orbscan II e paquímetro ultra-sônico
}

\author{
Reliability of corneal thickness measurementsby Orbscan II and ultrasound pachymeter
}

\author{
Adamo Lui Netto ${ }^{1}$ \\ João Baptista Nigro Santiago Malta² \\ Maurício Arruda Câmara Barros ${ }^{2}$ \\ Renato Giovedi Filho ${ }^{3}$ \\ Milton Ruiz Alves ${ }^{4}$
}

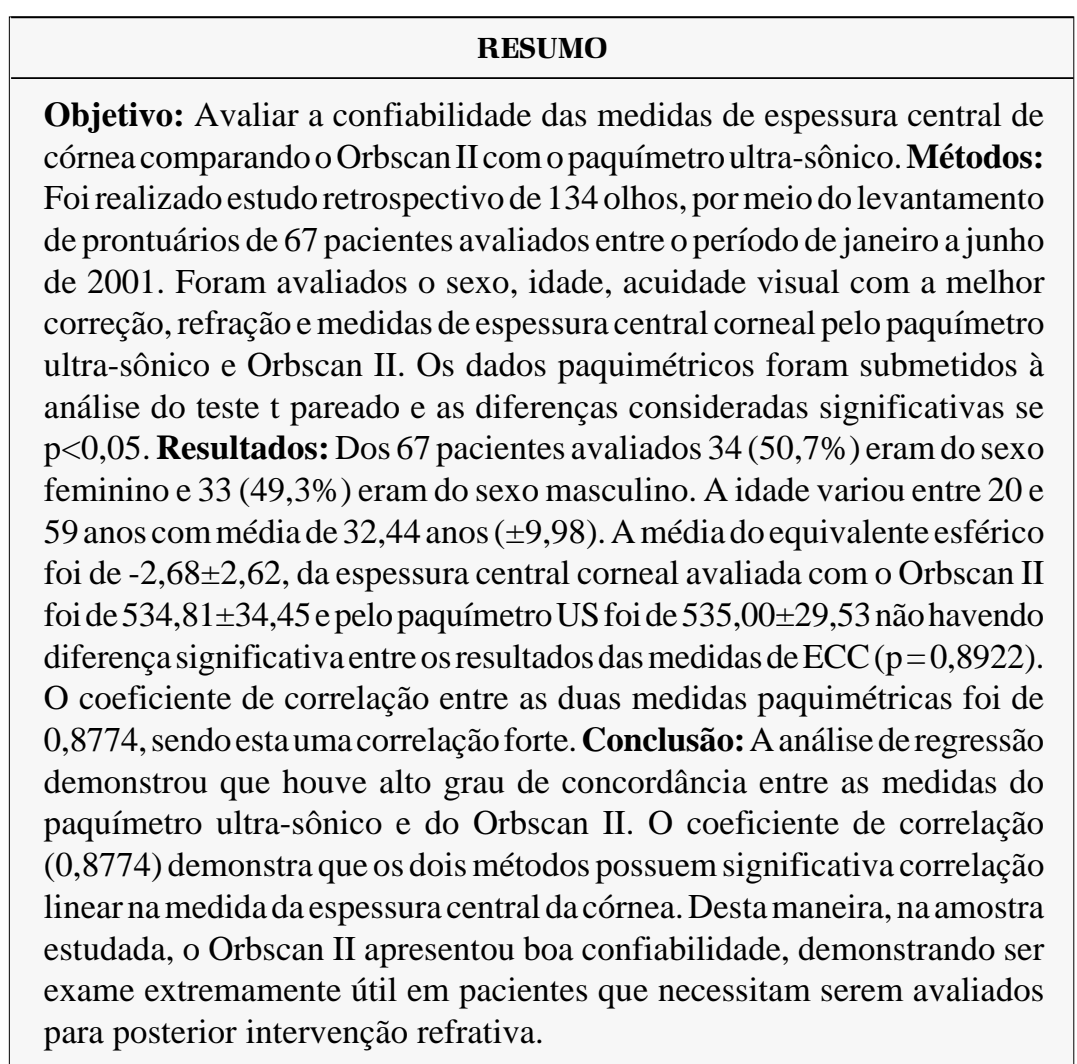

Descritores: Testes visuais/métodos; Córnea/anatomia \& histologia; Equipamentos de medição; Topografia da córnea; Confiabilidade da tecnologia; Erros de refração; Estudo comparativo
Trabalho realizado na Clínica particular do Dr. Adamo Lui Netto.

${ }^{1}$ Chefe do Setor de Lentes de contato do Departamento de Oftalmologia da Santa Casa de São Paulo.

${ }^{2}$ Residente do 2ํano do Departamento de Oftalmologia da Santa Casa de São Paulo.

${ }^{3}$ Chefe do Setor de Refração do Departamento de Oftalmologia da Santa Casa de São Paulo.

${ }^{4}$ Professor Livre Docente da Clínica Oftalmológica da Faculdade de Medicina da Universidade de São Paulo.

Endereço para correspondência: João Baptista N. S. Malta, Av. Comendador Adibo Ares, 1590 - São Paulo (SP) - CEP 05613-001

E-mail: jbmalta@hotmail.com

Recebido para publicação em 26.09.2003

Versão revisada recebida em 16.06.2004

Aprovação em 10.11.2004

\section{INTRODUÇ̃̃̃O}

Atualmente a espessura corneal central (ECC) apresenta importância significativa em relação a uma série de doenças oculares, como distrofias ectásicas, olho seco, glaucoma e diabetes mellitus ${ }^{(1)}$.

A paquimetria é considerada também importante fator para o acompanhamento de indivíduos usuários crônicos de lentes de contato e para aqueles que irão ser submetidos a procedimentos refrativos ${ }^{(2-4)}$.

A determinação dos níveis de edema da córnea pode ser parâmetro para permitir que o usuário continue ou não utilizando lentes de contato $^{(5)}$.

A ECC é um fator essencial para avaliar edema corneal subclínico e a função da bomba epitelial ${ }^{(1)}$. Um estudo publicado demonstrou haver variação diurna da ECC relacionada à hidratação da córnea de aproximadamente $9 \mu \mathrm{m}^{(6)}$. 
Além disso, estudos demonstram que variações da ECC podem causar distorções nas medidas da pressão intra-ocular (PIO) sendo que estas podem ser falsamente elevadas em indivíduos com espessura maior que $600 \mu \mathrm{m}$ e falsamente reduzidas em indivíduos com córneas mais finas (menor que $500 \mu \mathrm{m})^{(7)}$.

Diversos aparelhos são capazes de realizar medidas paquimétricas: paquímetro óptico, paquímetro ultra-sônico, Orbscan II, microscópio especular de contato e não contato, tomografia de coerência óptica, interferometria a laser, microscopia confocal e biomicroscopia ultra-sônica ${ }^{(1-2,8-13)}$.

O paquímetro ultra-sônico, durante anos, foi considerado o exame padrão para a $\mathrm{ECC}^{(14)}$. As medidas relacionam-se com a velocidade que o ultra-som atravessa a espessura corneal ${ }^{(10)}$.

O Orbscan II é o primeiro instrumento que pode proporcionar simultaneamente a topografia e a espessura corneal. A espessura corneal é determinada pela substração da superfície de elevação anterior da posterior ${ }^{(5)}$.

Além disso, o Orbscan II possui outras funções como, avaliação da profundidade da câmara anterior e medida de diâmetro corneal ${ }^{(14)}$.

As medidas paquimétricas em muitos casos são repetidas diversas vezes e nem sempre pela mesma pessoa. Por este motivo a precisão e a confiabilidade do aparelho independente do operador são fundamentais ${ }^{(2)}$.

O objetivo deste estudo é avaliar a confiabilidade das medidas de espessura corneal central com o Obscan II e o paquímetro ultra-sônico.

\section{MÉTODOS}

Foi realizado estudo retrospectivo por meio do levantamento de prontuários da clínica particular de um dos autores.

Os prontuários de 67 pacientes que seriam submetidos à cirurgia refrativa foram selecionados de maneira aleatória. Os pacientes foram avaliados entre o período de janeiro a junho de 2001.

Os critérios de inclusão no estudo foram: ausência de anormalidade no exame oftalmológico e preenchimento completo dos prontuários.

Foram avaliados o sexo, idade, acuidade visual com a melhor correção, refração e medidas de espessura central corneal pelo paquímetro ultra-sônico (Storz 6 F 2000 - Corneo Scan) e Orbscan II (Bausch \& Lomb ${ }^{\circledR}$, Rochester, New York, USA).

Dos 67 pacientes avaliados $34(50,7 \%)$ eram do sexo feminino e $33(49,3 \%)$ eram do sexo masculino. A idade variou entre 20 e 59 anos com média de 32,44 anos $( \pm 9,98)$.

A acuidade visual com a melhor correção dos 134 olhos variou entre $0,8(20 / 25)$ e $1,0(20 / 20)$ com média de $0,96( \pm 0,05)$.

As medidas de espessura central foram avaliadas em todos os pacientes no mesmo período do dia (entre 16 e $18 \mathrm{~h}$ ) e em ambos os olhos.

Primeiro, foram realizadas duas medidas com o Orbscan II (instrumento de não contato) e a menor medida foi considerada. A seguir, foram realizadas três medidas da ECC com o instru- mento de contato (paquímetro ultra-sônico) e a média foi anotada. Para tanto foi instilada uma gota de colírio anestésico $\left(\right.$ Anestalcon $\left.{ }^{\circledR}\right)$ em cada olho e o paciente foi colocado em decúbito dorsal horizontal, sendo a sonda posicionada perpendicularmente ao centro da córnea.

O fator acústico de correção do Orbscan II utilizado neste estudo foi de 0,92 .

Os resultados foram analisados por meio do GraphPad Instat Software. Os dados sobre idade e refração foram submetidos à análise do teste $\mathrm{t}$ simples. Os dados paquimétricos foram submetidos à análise do teste t pareado e considerados significativos se $p<0,05$. O coeficiente de correlação foi calculado empregando-se a da fórmula de Pearson.

\section{RESULTADOS}

Foram avaliados ambos os olhos dos 67 pacientes num total de 134 olhos.

A tabela 1 mostra os resultados da ECC avaliados pelo método do paquímetro ultra-sônico e Orbscan II, assim como o equivalente esférico dos 134 olhos.

$\mathrm{O}$ teste $\mathrm{t}$ pareado foi utilizado para analisar os resultados da ECC com os dois métodos paquimétricos e demonstrou que não houve diferença significativa entre os métodos $(\mathrm{p}=0,89)$.

O coeficiente de correlação entre as duas medidas paquimétricas foi de 0,8774 , sendo esta uma correlação forte. O gráfico 1 evidencia estes resultados.

\section{DISCUSSÃO}

$\mathrm{Na}$ avaliação da ECC, o principal problema encontrado nas diferentes técnicas paquimétricas, realizadas com diferentes aparelhos, é a discrepância dos resultados ${ }^{(10)}$.

A literatura mostra contradição nos resultados encontrados por diferentes autores, na medida da ECC pelo Orbscan II e pelo paquímetro ultra-sônico (Tabela 2).

Existem diversas razões para explicar os resultados discrepantes e os valores superestimados da ECC demonstrado pelo Orbscan II.

As diferenças existentes entre os dois aparelhos resultam dos diferentes princípios de funcionamento. O Orbscan II, que funciona como paquímetro de não contato, mede a espessura corneal entre o ar, interface do filme lacrimal e superfície posterior da córnea ${ }^{(1)}$.

\begin{tabular}{|c|c|c|c|c|}
\hline & & & & $n=134$ \\
\hline & Média \pm DP & EP & Variação & Mediana \\
\hline ORB & $534,81 \pm 34,45$ & 2,97 & $431-621$ & 537 \\
\hline US & $535,00 \pm 29,53$ & 2,55 & $480-607$ & 533 \\
\hline Eq. Esf & $-2,68 \pm 2,62$ & & $-8,75-4,50$ & $-3,00$ \\
\hline
\end{tabular}




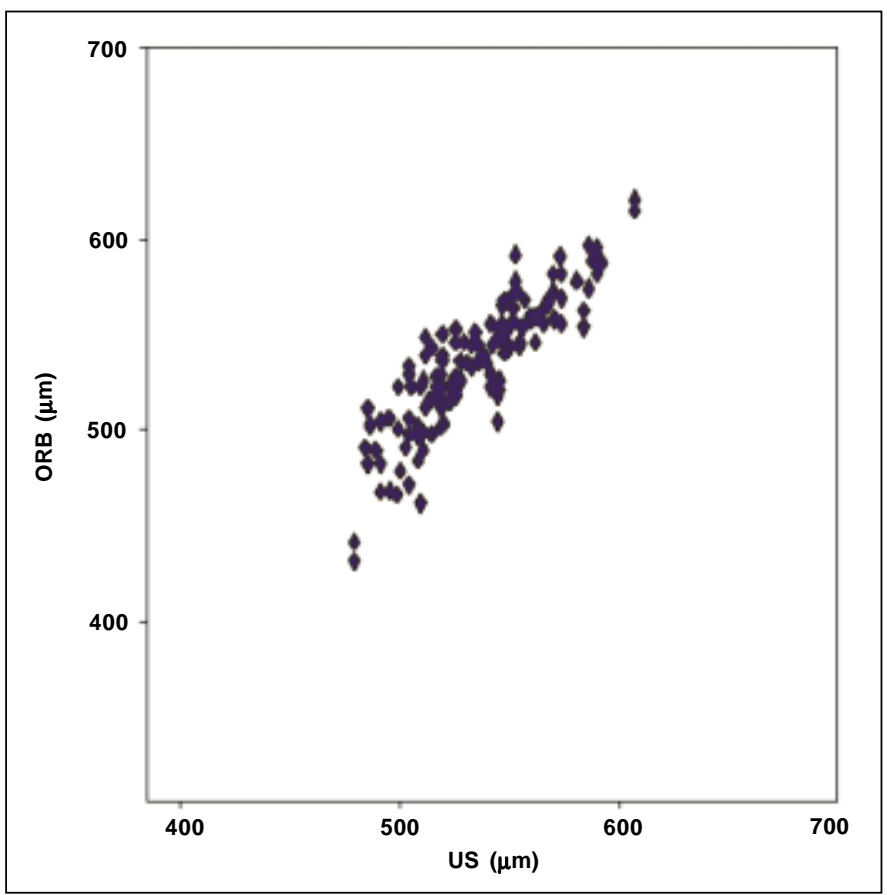

Gráfico 1 - Diagrama de dispersão entre medidas da espessura central da córnea (ECC) de 134 olhos com o Orbscan (ORB) e Paquímetro ultra sônico (US)

\begin{tabular}{|c|c|c|c|}
\hline & Orbscan & Ultra-som & Diferença \\
\hline Chakrabarti(20) & $566,0 \pm 40,7$ & $538,0 \pm 36,0$ & 28,00 \\
\hline Fakhry ${ }^{(2)}$ & $529,5 \pm 54,5$ & $527,0 \pm 53,0$ & 1,85 \\
\hline Marsich(5) & $596,0 \pm 40,0$ & $542,0 \pm 33,0$ & 54,00 \\
\hline Modis $^{(1)}$ & $602,0 \pm 59,0$ & $580,0 \pm 43,0$ & 22,00 \\
\hline Prisant $^{(19)}$ & $546,0 \pm 40,0$ & $531,0 \pm 39,0$ & 15,00 \\
\hline
\end{tabular}

Nos paquímetros ultra-sônicos, o ponto exato de reflexão corneal posterior não é certo, podendo ser deslocado da membrana de Descemet até a câmara anterior ${ }^{(15)}$. Além disso, a sonda do ultra-som pode ser facilmente deslocada de 7 a $40 \mu \mathrm{m}$ para dentro da espessura do filme lacrimal e pode ocasionar desepitelização durante o exame ${ }^{(15-16)}$.

A indentação causada por estes paquímetros poderia subestimar os valores reais paquimétricos. Porém, autores observaram que a ECC aferida pelo paquímetro ultra-sônico não é modificada pela indentação causada pela sonda do aparelho ${ }^{(17)}$.

Além disso, um dos principais motivos de flutuação das medidas do paquímetro ultra-sônico é a angulação da sonda em relação ao epitélio da córnea ${ }^{(5)}$.

Outros autores sugerem que o Orbscan II pode não apenas medir a ECC assim como o filme lacrimal que recobre a superfície anterior da córnea, que pode ter até $40 \mu \mathrm{m}$ de espessura( ${ }^{(16)}$.

No presente estudo não houve diferença estatisticamente significante entre os resultados da ECC avaliada pelo Obscan II e pelo paquímetro US.
As possíveis explicações para tal fato são que todos os exames foram realizados no mesmo período do dia, evitando a variação de espessura de córnea e por um mesmo examinador ${ }^{(6)}$. Talvez o modelo do paquímetro ultra-sônico utilizado possa ter influência nos resultados apresentados.

Tanto o Orbscan II como o paquímetro ultra-sônico possuem vantagens e desvantagens.

O Orbscan II proporciona a grande vantagem de oferecer num mesmo exame a análise do mapa de elevação topográfica da córnea, avaliando simultaneamente a superfície corneal anterior e posterior, e medidas de paquimetria central, paracentral e periféricas, assim como o ponto de menor espessura, sendo este seu real diferencial tecnológico. Além disso, é um exame rápido e não invasivo ${ }^{(1,5,18-19)}$.

As principais desvantagens são seu alto custo, em torno de U\$30,000, e a impossibilidade de transporte para diferentes locais $^{(5)}$.

O paquímetro ultra-sônico apresenta custo reduzido quando comparado ao Orbscan II, em torno de U\$ 5,000. É o exame de eleição para medida da ECC, além de ser um aparelho portátil ${ }^{(5,10)}$.

Porém é exame de contato com possível risco de desepitelização e infecção iatrogênica, necessita de colírio anestésico, apresenta dificuldade para posicionar a sonda no ponto exato da córnea e apresenta resultados discrepantes quando realizado por diferentes observadores e por diferentes aparelhos ${ }^{(20)}$.

Neste estudo, a análise de regressão demonstrou que houve um alto grau de concordância entre as medidas do paquímetro ultra-sônico e do Orbscan II.

O coeficiente de correlação $(0,87)$ demonstra que os dois métodos possuem significativa correlação linear na medida da espessura central da córnea.

\section{CONCLUSÕES}

No presente estudo o paquímetro ultra-sônico e o Orbscan II apresentaram boa confiabilidade, demonstrando ser exames de grande utilidade na aferição da ECC em pacientes que necessitam serem avaliados para posterior intervenção refrativa.

\section{ABSTRACT}

Purpose: To access the reliability of corneal thickness measurements by Orbscan II and ultrasound pachymeter. Methods: A retrospective study of 134 eyes from 67 normal subjects between January and June 2001 was obtained. Sex, age, visual acuity, refractive error and corneal thickness measurements by Orbscan II and ultrasound pachymetry were evaluated. All results were analyzed by the paired t test $(\mathrm{p}<0.05)$. Results: Sixty-seven subjects, 34 (50.7\%) female and 33 (49.3\%) male, with a mean age of $32.44 \pm 9.98$ were evaluated. The mean of spherical equivalent was $-2.68 \pm 2.62$. The mean corneal thickness was $534.81 \pm 34.45$ with the Orbscan II system and 
$535.00 \pm 29.53$ with the ultrasound pachymeter, values that were not significantly different $(\mathrm{p}=0.8922)$. The correlation coefficient between both instruments was 0.8774 , and it is effective. Conclusions: Both methods are similar showing good correlation. In this study, the Orbscan II showed reliability, and this tool is extremely useful to evaluate subjects regarding refractive surgery.

Keywords: Vision tests/methods; Cornea/anatomy \& histology; Measurement equipment; Corneal topography; Technology realibility; Refractive errors; Comparative study

\section{REFERÊNCIAS}

1. Modis L Jr, Langenbucher A, Seitz B. Scanning-slit and specular microscopic pachymetry in comparison with ultrasonic determination of corneal thickness. Cornea. 2001;20(7):711-4.

2. Fakhry MA, Artola A, Belda JI, Ayala MJ, Alio JL. Comparison of corneal pachymetry using ultrasound and Orbscan II. J Cataract Refract Surg. 2002;28(2):248-52.

3. Paranhos JFS, Toledo Netto L, Nassaralla BRA. Paquimetria esperada X obtida após LASIK para miopia. Arq Bras Oftalmol. 2001;64(3):185-7.

4. Tanaka HM, Mori ES, Maia NCF, Juliano Y, Campos M. Espessura corneana na alta miopia. Arq Bras Oftalmol. 1996;59(3):295-8.

5. Marsich MW, Bullimore MA. The repeatability of corneal thickness measures. Cornea. 2000;19(6):792-5.

6. Lattimore MR Jr, Kaupp S, Schallhorn S, Lewis R 4th. Orbscan pachymetry: implications of a repeated measures and diurnal variation analysis. Ophthalmology. 1999;106(5):977-81.

7. Sakata K, Figueira ALM, Guimarães ACP, Schmitt AJ, Scapucin L, Barros LGR, et al. Estudo da correlação entre pressão intra-ocular e espessura corneana central (projeto glaucoma). Arq Bras Oftalmol. 2000;63(5)355-8.
8. Bechmann M, Thiel MJ, Neubauer AS, Ullrich S, Ludwig K, Kenyon KR, et al. Central corneal thickness measurement with a retinal optical coherence tomography device versus standard ultrasonic pachymetry. Cornea. 2001;20(1):50-4.

9. Bovelle R, Kaufman SC, Thompson HW, Hamano H. Corneal thickness measurements with the Topcon SP-2000P specular microscope and an ultrasound pachymeter. Arch Ophthalmol. 1999;117(7):868-70.

10. Giraldez Fernandez MJ, Diaz Rey A, Cervino A, Yebra-Pimentel E. A comparison of two pachymetric systems: slit-scanning and ultrasonic. CLAO J. 2002;28(4):221-3.

11. Muscat S, McKay N, Parks S, Kemp E, Keating D. Repeatability and reproducibility of corneal thickness measurements by optical coherence tomography. Invest Ophthalmol Vis Sci. 2002;43(6):1791-5.

12. Rainer G, Petternel V, Findl O, Schmetterer L, Skorpik C, Luksch A, et al. Comparison of ultrasound pachymetry and partial coherence interferometry in the measurement of central corneal thickness. J Cataract Refract Surg. 2002;28 (12):2142-5.

13. Wirbelauer C, Scholz C, Hoerauf H, Pham DT, Laqua H, Birngruber R. Noncontact corneal pachymetry with slit lamp-adapted optical coherence tomography. Am J Ophthalmol. 2002;133(4):444-50.

14. Iskander NG, Anderson Penno E, Peters NT, Gimbel HV, Ferensowicz M. Accuracy of Orbscan pachymetry measurements and DHG ultrasound pachymetry in primary laser in situ keratomileusis and LASIK enhancement procedures. J Cataract Refract Surg. 2001;27(5):681-5.

15. Nissen J, Hjortdal JO, Ehlers N, Frost-Larsen K, Sorensen T. A clinical comparison of optical and ultrasonic pachymetry. Acta Ophthalmol (Copenh). 1991;69(5):659-63.

16. Liu Z, Huang AJ, Pflugfelder SC. Evaluation of corneal thickness and topography in normal eyes using the Orbscan corneal topography system. $\mathrm{Br} \mathrm{J}$ Ophthalmol. 1999;83(7):774-8.

17. Solomon O D. Corneal indentation during ultrasonic pachymetry. Cornea. 1999;18(2):214-5.

18. Boscia F, La Tegola MG, Alessio G, Sborgia C. Accuracy of Orbscan optical pachymetry in corneas with haze. J Cataract Refract Surg. 2002;28(2):253-8.

19. Prisant O, Calderon N, Chastang P, Gatinel D, Hoang-Xuan T. Reliability of pachymetric measurements using orbscan after excimer refractive surgery. Ophthalmology. 2003;110(3):511-5.

20. Chakrabarti HS, Craig JP, Brahma A, Malik TY, McGhee CN. Comparison of corneal thickness measurements using ultrasound and Orbscan slit-scanning topography in normal and post-LASIK eyes. J Cataract Refract Surg. 2001;27 (11):1823-8.

\section{Congresso da Sociedade Caipira de Oftalmologia \\ IV Simpósio da Sociedade de Enfermagem em Oftalmologia}

\section{0 a 3 de Julho de 2005 \\ Mendes Convention - Santos - SP}

IN FO RMAÇÕ ES: CENACON - Centro Nacional de Congressos

Tel: (17) 235-7017

E-mail: cenacon@ cenacon.com.br 